\title{
Complement activation on immunoglobulin G- coated hydrophobic surfaces enhances the release of oxygen radicals from neutrophils through an actin- dependent mechanism
}

\author{
Jonas Wetterö, Torbjörn Bengtsson and P Tengvall
}

\section{Linköping University Post Print}

N.B.: When citing this work, cite the original article.

This is the authors' version of the final publication:

Jonas Wetterö, Torbjörn Bengtsson and $\mathrm{P}$ Tengvall, Complement activation on immunoglobulin G-coated hydrophobic surfaces enhances the release of oxygen radicals from neutrophils through an actin- dependent mechanism, 2000, Journal of Biomedical Materials Research, (51), 4, 742-751.

http://dx.doi.org/10.1002/1097-4636(20000915)51:4\&lt;742::AID-JBM24\&gt;3.0.CO;2-D

Copyright: Wiley

Postprint available at: Linköping University Electronic Press

http://urn.kb.se/resolve?urn=urn:nbn:se:liu:diva-25790 


\section{Complement activation on immunoglobulin G-coated hydrophobic surfaces enhances the release of oxygen radicals from neutrophils through an actin-dependent mechanism ${ }^{3}$}

\section{Jonas Wetterö $^{\mathrm{a}, \mathrm{b}}$, Torbjörn Bengtsson ${ }^{\mathrm{b}}$ and Pentti Tengvall ${ }^{\mathrm{a}}$}

${ }^{a}$ Laboratory of Applied Physics, Department of Physics and Measurement Technology, Linköping University, SE-581 83 Linköping, Sweden

${ }^{b}$ Division of Medical Microbiology, Department of Health and Environment, Faculty of Health Sciences, Linköping University, SE-581 85 Linköping, Sweden

Correspondence to: Jonas Wetterö (e-mail: jonwe@ifm.liu.se)

Key Words: neutrophil respiratory burst, IgG, complement, wettability, actin cytoskeleton

\footnotetext{
${ }^{3}$ No benefit of any kind will be received either directly or indirectly by the authors
} 
Abstract: Neutrophil granulocytes are among the first cells to encounter a plasma protein-coated implant and may through frustrated phagocytosis release toxic oxidative species. We utilized two model surfaces, hydrophobic and hydrophilic glass, to investigate the effects of plasma immunoglobulin G (IgG) - complement interactions for neutrophil adhesion and respiratory burst. The respiratory burst was measured with luminol-amplified chemiluminescence (CL) and cell adhesion was determined by labeling neutrophils with 2', 7'-bis-(carboxy-ethyl)-5(6)carboxyfluorescein (BCECF). We demonstrate that the IgG-triggered neutrophil adhesion and oxygen radical production is augmented in the presence of normal human serum (NHS), in particular on hydrophobic surfaces,indicating that complement factors enhance the neutrophil activation. We propose that the complement factors C3, C5a and C1q are especially important for this amplification, but factor B is probably not. Disturbance of the actin filament dynamics with cytochalasin B or jasplakinolide blocked the neutrophil radical generation on all surfaces. However, these drugs did not affect the number of adherent neutrophils. We suggest that there is a synergistic interaction between adsorbed IgG, and the complement system , which amplifies the neutrophil acute inflammatory responses through a dynamic actin cytoskeleton on synthetic surfaces. 


\section{INTRODUCTION}

An artificial surface that encounters blood becomes coated with plasma proteins e.g. fibrinogen, albumin and immunoglobulin G (IgG) within seconds. ${ }^{1}$ The role of plasma proteins in the host recognition of implants is poorly characterized, but it is evident that the surface chemistry and wettability strongly influence the composition of the adsorbed layer. Vroman exchange-like phenomena are reported at hydrophilic surfaces ${ }^{2}$ and fibrin/fibrinogen appears to be of great importance in mediating the acute inflammatory response at hydrophobic surfaces. ${ }^{3}$ Albumin is suggested to blunt the inflammatory response ${ }^{4}$ and adsorbed IgG is known to activate and recruit blood cells in vitro ${ }^{4,5}$ and in vivo. ${ }^{6}$

The activation of complement during blood-biomaterial interactions is a clinically observed phenomenon, ${ }^{7-11}$ although the mechanisms are not fully understood. Probably both the classical and the alternative complement pathways are involved. In brief, the classical activation is initiated by immunoglobulins and complement factor 1q (C1q) leading to deposition of C3b and activation on the target surface. The alternative complement activation is triggered by anon-specific binding of C3b. Both pathways serve to form C3-convertases that cleave C3 to form C3a and C3b. ${ }^{12}$ The binding of complement to artificial surfaces may also be affected by other plasma proteins. ${ }^{13}$

Neutrophils can be found on artificial surfaces within minutes of blood contact. ${ }^{14-16}$ The cell migration is initiated by a local release of chemotactic stimuli, for instance complement components in a damaged tissue. The neutrophil phagocytosis of a prey involves fixation of the target to the neutrophil surface. Most often this is accomplished through the interaction between opsonizing complement residues on the target surface and complement receptors on the neutrophil plasma membrane. Neutrophils carry complement receptors (CR1 and CR3) that bind C1q, C3b and iC3b. However, the 
fixation is not complete unless a second activation occurs from either bacterial carbohydrates or opsonising IgG (ligand for neutrophil Fc $\gamma$-receptors II and III). ${ }^{17}$ Apart from enhancing the neutrophil adhesiveness and the phagocytic behavior on biomaterial surfaces, ${ }^{18}$ adsorbed IgG induces the neutrophil generation of oxygen free radicals. ${ }^{5}$ Furthermore, immobilized IgG has been suggested to activate C3 in serum and, accordingly, increase the C3b and iC3b depositions onto both hydrophobic and hydrophilic surfaces. ${ }^{19}$

The microbicidal activity of neutrophils can be divided into mechanisms dependent or independent on oxygen. ${ }^{20,}{ }^{21}$ The oxygen-dependent mechanisms consume oxygen as an electron acceptor in a reaction initiated by the activation of a multicomponent electron transfer system, the NADPHoxidase. This way relatively atoxic superoxide anions are produced which can be dismuted by superoxide dismutase (SOD) to antimicrobical hydrogen peroxide. In parallel with the generation of toxic oxidative products, protective antioxidant systems are activated. For instance, catalase produces water and oxygen from hydrogen peroxide. The neutrophil respiratory burst is often studied with luminol-amplified chemiluminescence (CL). ${ }^{22}$

The neutrophil spreading and migration over a surface requires rearrangements of the actin cytoskeleton, including reversible transformations between globular and filamentous (F) actin. Actin filaments organized in adhesion sites (focal contacts) and pseudopodia are considered to play a critical role in neutrophil behavior by regulating the dynamic interactions between $\beta_{2}$-integrins and extracellular matrix proteins. Several findings also indicate that secretion of hydrolytic enzymes and production of oxygen metabolites are directly regulated by the actin filament system. ${ }^{23-25}$ In support, an association between components of the NADPH-oxidase, $\beta_{2}$-integrins (e.g. CR3) and actin cytoskeletal structures has been reported. ${ }^{26,27}$ 
In the present study we used a well defined clinically relevant biomaterial wettability model-system ${ }^{5}$, 28-30 in order to elucidate the effects of IgG on neutrophil respiratory burst and adhesion and its correlation to complement activation and actin rearrangements.

\section{MATERIALS AND METHODS}

\section{Materials}

All reagents were of analytical grade and purchased from Sigma Chemical Co. (St. Louis, MO, USA) if nothing else is denoted. Cell separation products dextran and Ficoll-Paque were from Pharmacia Fine Chemicals (Uppsala, Sweden). Sodium-metrizoate was delivered from Nycomed Pharma (Oslo, Norway). CL reagents horseradish peroxidase, SOD and catalase were from Boehringer Mannheim GmbH (Mannheim, Germany). Normal human IgG (Gammaglobulin 165 mg/ml) was from KabiPharmacia (Uppsala, Sweden). In the CL assay four ml disposable polypropylene tubes (Sarstedt GmbH, Nümbrecht, Germany) and 40 x $8 \mathrm{~mm}$ glass test tubes with a high $\mathrm{SiO}_{2}$ content (Assistent KHG, Sondheim-Rhön, Germany) were used. Complement-depleted sera and purified complement components were from Quidel Corp. (San Diego, CA, USA). Giemsa staining solution was from Merck (Darmstadt, Germany). Bodipy-phallacidin and jasplakinolide were purchased from Molecular Probes Inc. (Eugene, OR, USA). The antibodies M 0741 (Monoclonal Mouse Anti-Human C3bi

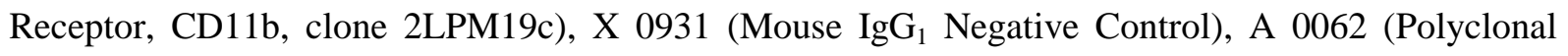
Rabbit Anti-Human C3c Complement) and A 424 (Polyclonal Rabbit Anti-Human IgG, Specific for $\gamma$ Chains) were from Dakopatts (Glostrup, Denmark). Azide free monoclonal F(ab’) $)_{2}: \mathrm{s}$ RDI-023-301 (mouse anti-human CD 32, clone IV.3), RDI-028-201X (mouse anti-human CD 16, clone 3G8) and mouse IgG $_{2}$ isotype control antibody RDI-MSIGG2A-X (clone G155-178) were from Research Diagnostics, Inc. (Flanders, NJ, USA). Monoclonal mouse anti human CD88 (MCA1283XZ, clone S5/1) came from Serotec (Oxford, England). Purified (>80\%) P1-peptide (HKYGWTVFQKKRLDGSV-OH) was from Research Genetics (Huntsville, AL, USA). 2', 7’-bis- 
(carboxy-ethyl)-5(6)-carboxyfluorescein pentaacetoxymethyl ester (BCECF-AM) was from Calbiochem (La Jolla, CA, USA).

\section{Neutrophils}

Peripheral blood was drawn daily from apparently healthy volunteers into heparinized tubes. The erythrocytes were removed by dextran-sodium metrizoate (2:1) sedimentation at $1 \mathrm{x} g$, followed by 35 seconds of hypotonic lysis in ice-cold distilled water. The neutrophils were then separated from monocytes, lymphocytes and platelets by Ficoll-Paque centrifugation $\left(400 \mathrm{x} g, 4^{\circ} \mathrm{C}\right)$ and washed twice (200 x $\mathrm{g}, 4^{\circ} \mathrm{C}$ ) in Krebs-Ringer phosphate buffer, $\mathrm{pH}$ 7.3, supplemented with $1.5 \mathrm{mM} \mathrm{Mg}{ }^{2+}$ and $5 \mathrm{mM}$ glucose. ${ }^{31}$ The washed neutrophils were then resuspended in the previous buffer with an addition of 1 $\mathrm{mM} \mathrm{Ca}^{2+}(\mathrm{KRG})$ and counted in a Coulter Counter (Coulter Electronics, Ltd., England). The isolated neutrophils were kept on ice until the experiments were performed within a few hours after the preparation. Morphological studies showed no signs of cell activation due to the preparation procedure, a high cell viability and very low contamination of other blood cells. Control experiments were performed with neutrophils isolated via alternative preparation techniques from citrated whole blood and buffy coats, as well as by using diluted heparinized whole blood.

\section{Sera}

Normal human serum (NHS) was prepared by clotting and centrifugation of fresh whole blood from three AB-positive donors at room temperature. In some experiments, the NHS was decomplemented by heat-inactivation for 30 minutes at $56^{\circ} \mathrm{C}$. Both types of sera were filtered through sterile $0.22 \mu \mathrm{m}$ Millipore Millex-GV filters in order to remove cellular debris. Commercially decomplemented NHS depleted on or deficient ${ }^{32}$ in complement components C1q, C3 and B, respectively, were also used. The functional activity of these was restored by addition of the purified components. All types of sera were frozen below $-70^{\circ} \mathrm{C}$ immediately after preparation. Most often, a final serum concentration of 5\% (v/v) was used. Control experiments with heparinized or citrated plasma were also performed. 
Importantly, frozen NHS elicited the same CL response as the freshly prepared, and the sterile filtration of the NHS did not interfere with the study.

\section{Hydrophobic and hydrophilic surfaces}

Glass test tubes were cleaned for five minutes at $80^{\circ} \mathrm{C}$ in a solution consisting of five parts of distilled water, one part of hydrogen peroxide $(30 \% \mathrm{v} / \mathrm{v})$ and one part of $\mathrm{NH}_{4} \mathrm{OH}(25 \% \mathrm{v} / \mathrm{v})$. After repeated rinsing in distilled water, the tubes were washed for five minutes at $80^{\circ} \mathrm{C}$ in another solution composed of six parts of distilled water, one part of hydrogen peroxide $(30 \% \mathrm{v} / \mathrm{v})$ and one part of hydrochloric acid $(37 \% \mathrm{v} / \mathrm{v})$. This procedure covers the surface with a thin, hydrated, layer of $\mathrm{SiO}_{2}$ and generates a water/surface advancing contact angle $\theta_{\mathrm{w}}<10^{\circ}$ (measured with a Ramé-Hart NRL Model 100 goniometer). Some of the hydrophilic tubes were saved and stored in an acid solution. The other tubes were washed in distilled water, technically pure ethanol and trichlorethylene, and methylated through five minutes of incubation at room temperature in trichlorethylene with $1 \%(\mathrm{v} / \mathrm{v})$ dichlorodimethylsilane added. Traces of silane was removed through excessive rinsing in ethanol, trichlorethylene and, finally, ethanol. The hydrophobic preparations were stored in ethanol for not more than a week and the hydrophobicity was continuously checked $\left(\theta_{\mathrm{w}}>90^{\circ}\right)$.

\section{Coating with IgG}

A stock solution of $10 \mathrm{mg}$ IgG per ml was prepared in phosphate buffered saline, $\mathrm{pH} 7.3$ (PBS). The normal human IgG used was derived from the plasma pool of approximately 10,000 European blood donors and should have an immunoglobulin A contamination of no more than $0.05 \%$. The purity of this product was checked via reduced SDS-PAGE. Hydrophobic and hydrophilic tubes were washed twice in PBS, then $1 \mathrm{mg} / \mathrm{ml}$ of IgG in PBS was incubated in the test tubes for 30 minutes at room temperature. Before the final sample solutions were added, the tubes were rinsed twice in PBS. By this procedure, a monolayer of 
IgG was adsorbed to the glass surfaces. The antigenicity was controlled in experiments using similar silicon surfaces, antibody A 424 and single wavelength ellipsometry in air (on a Rudolf Research AutoEl, NJ, USA, with a HeNe $\lambda=6328 \AA$ laser) in order to determine the thickness of the adsorbed monolayer (approximately $20 \AA ̊$ ). In some experiments, surfaces were coated with human serum albumin as a negative reference to IgG (approximately $12 \AA$ ).

\section{Neutrophil radical production}

Glass test tubes (IgG- or non-coated) with total sample volumes of $0.5 \mathrm{ml}$ in KRG were placed inside four ml polypropylene tubes. ${ }^{5}$ NHS was added to some samples, and all solutions were mixed and allowed to equilibrate at $37^{\circ} \mathrm{C}$ for a couple of minutes. After this, CL reagents and neutrophils were added. Luminol (5-amino-2,3-dihydro-1,4-phtalazinedione, $50 \mu \mathrm{M}$ ) and extra peroxidase (horseradish peroxidase, $4 \mathrm{U} / \mathrm{ml}$ ) were used for the determination of the total oxidase activity. The intracellular response was quantified using superoxide dismutase $(200 \mathrm{U} / \mathrm{ml})$ and catalase $(2,000 \mathrm{U} / \mathrm{ml})$ instead of extra peroxidase. The CL was registered for half an hour at $37^{\circ} \mathrm{C}$ in a just calibrated six channel Biolumat LB 9505 C from Berthold Co. (Wildbaden, Germany). The actin polymerization inhibitor cytochalasin B (12,5 $\mu \mathrm{g} / \mathrm{ml})$, or stimulator jasplakinolide (0.1-10 $\mu \mathrm{M})$, was added to, or pre-incubated with, the sample solutions in some experiments. In other experiments, the neutrophils were incubated with the CR3-blocking ${ }^{33,}{ }^{34}$ antibody M 0741, or with the P1-peptide $(100 \mu \mathrm{M})$, using the X 0931 antibody as a negative control. To elucidate a possible priming role of the anaphylatoxin C5a, the neutrophil C5a-receptor (CD88) was blocked with the azide free MCA 1283XZ antibody and compared with the response from cells treated with the control antibody RDI-MSIGG2A-X.

\section{Adhesion, morphology and F-actin distribution}

The number of adhered neutrophils was determined after 30 minutes of incubation inside the MultiBiolumat using several methods. First, the number of cells remaining in the cell was counted using a Coulter Counter ZM Channelyser 256 (Coulter-Electronics, Luton, UK). Secondly , neutrophils were fixed on slides and stained, counted and studied morphologically. The cells were fixed for 15 minutes in methanol for nuclear staining and incubated for 15 minutes in a Giemsa/methanol $(4+25$ parts, 
$\mathrm{v} / \mathrm{v}$ ) solution on ice, after which they were viewed by light microscopy. F-actin was stained and studied in a fluorescence microscope after 30 minutes of fixation in ice-cold paraformaldehyde (4\%) and 30 minutes incubation in a mixture of bodipy-phallacidin $(0.6 \mu \mathrm{g} / \mathrm{ml})$ and lysophosphatidylcholine $(100 \mu \mathrm{g} / \mathrm{ml})$ in PBS protected from light. ${ }^{35}$ Bodipy-Phallacidin stained samples were also documented with a Sarastro 1000 confocal laser scanning microscope from Molecular Dynamics (Sunnyvale, CA, USA). This microscope had a Nikon x 100 (1.4 N.A.) objective and the samples were excited at $488 \mathrm{~nm}$ by the Argon laser. The laser intensity and the photomultiplier voltage were kept constant throughout the photo sessions. As a third approach, the neutrophils were labeled with $4 \mu \mathrm{M}$ BCECF-AM (40 minutes in $37^{\circ} \mathrm{C}$ ) and washed twice in KRG (200 x g, $4^{\circ} \mathrm{C}$ ). The labeled cells were allowed to adhere to the surfaces, non-adherent cells were rinsed off and the remaining neutrophils were lysed with $0.5 \%(\mathrm{v} / \mathrm{v})$ Triton X-100. ${ }^{36,37}$ The number of adhered neutrophils were then calculated by excitation of the samples at $\lambda=439 \mathrm{~nm}$ and the fluorescence was measured at $\lambda=505 \mathrm{~nm}$ in a Perkin-Elmer LS-3B spectrofluorometer (Perkin-Elmer Ltd., Beaconsfield, Buckinghamshire, England). The fluorescence emanating from the adhered neutrophils was divided with the total fluorescence from free and surface-associated cells. The autofluorescence from non-labeled cells was routinely subtracted.

\section{Statistics}

Data from the experiments are expressed as the mean values \pm standard errors of the means (SEM). Students two-tailed T-test for paired observations was used for the comparison between activated cells and control cells. Differences with $\mathrm{p}<0.05$ were regarded as significant.

\section{RESULTS}

\section{The effect of wettability on IgG immobilization and the neutrophil respiratory burst}

The exposure of neutrophils in KRG to pure hydrophilic glass rendered a more pronounced oxygen radical production than cells subjected to a hydrophobic surface. The average CL of neutrophils from 
19 donors run in duplicate was $1,78 \pm 0,12 \times 10^{8}$ counts per minute (CPM) on hydrophilic, and 1,36 \pm 0,11 x $10^{8}$ CPM on hydrophobic surfaces ( $<<0.001$ ). Pre-adsorbed IgG appeared to slightly enhance the respiratory burst on both types of surfaces, although the CL responses did not differ significantly from those of control cells (Figure 1). The CL responses, that included both extra- and intracellular oxygen radical production, were monophasic with a peak after approximately five minutes and declined within an hour. However, pre-coating of either surface with IgG augmented the radical production in whole blood (Table 1). Ellipsometric analysis using the antibody A 424 indicated that the IgG-monolayers were stable for days on hydrophobic silicon, whereas the antigenicity declined within hours after preparation on hydrophilic silicon. Furthermore, an increase of the IgGconcentration during the coating procedure did not further amplify the CLresponse.

\section{Complement activation on hydrophobic and hydrophilic glass}

The CL response in neutrophils interacting with IgG on hydrophobic or hydrophilic surfaces was augmented by the addition of NHS (Figure 1). The maximal CL-response was obtained with 5 to $10 \%(\mathrm{v} / \mathrm{v})$ NHS and the response declined within an hour. Studies with superoxide-dismutase and catalase indicated that mainly the extracellular release of oxygen metabolites was enhanced. The NHS-amplified CL response on the IgG-coated hydrophobic surfaces included a marked second phase after approximately 20 minutes. The second phase disappeared when NHS was heat-inactivated, p $<0.001$ (Figure 2), or when a C3-depleted serum was used. Furthermore, when the C3-depleted serum was reconstituted with C3, a response similar to that observed with NHS was restored (Figure 3). The "IgG/NHS-effect" could be quenched by rinsing the NHS preparations in KRG prior to the addition of cells. Ellipsometric investigation with antibody A 0062 on IgG-coated hydrophobic silicon surfaces revealed the deposition of C3 almost instantly after exposure to NHS, although no C3 was found when heat-inactivated NHS was used instead (not shown). Blocking of the neutrophil receptors

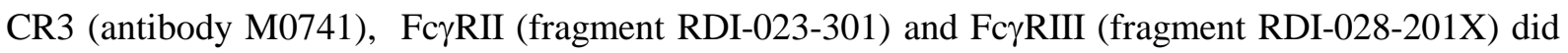
not alter the IgG-triggered CL response in the presence of NHS. However, blocking of CR3 with the P1-peptide, reduced the CL by approximately 50-75\%. The factor B-depleted serum behaved similar 
to NHS. Somewhat surprising, C1q-depleted serum was found to enhance the neutrophil respiratory burst biphasically when exposed to IgG-coated hydrophobic glass. The oxygen radical production was further amplified with the reconstitution of C1q to the depleted serum (Figure 4A). Blocking of CD88 reduced the overall "IgG/NHS-effect" by $22.7 \%(\mathrm{p}<0.05$, Figure 4B) and the IgG-triggered neutrophil response to heat-inactivated serum was lowered by $12.9 \%$ (not significant). Incubation with the negative control antibody showed no detectable effect on the CL-response.

In order to elucidate the possible participation of the most abundant plasma proteins, we added normal or heat-inactivated albumin, fibrinogen or IgG, respectively, into non- and IgG-coated hydrophobic glass tubes. Stimulatory effects similar to those of NHS were not obtained. A phenomenon similar to that elicited by NHS was observed when citrated platelet poor or platelet free plasma were exposed to IgG-coated hydrophobic glass.

The enhancement of the neutrophil oxygen radical production by the combination of IgG and NHS was much less pronounced on the hydrophilic compared to on the hydrophobic glass (Figure 2). The amplification was monophasic and disappeared when using heat-inactivated NHS instead (Figure 3). Interestingly, heat-inactivated NHS markedly increased the neutrophil generation of oxidative species on the hydrophobic surfaces, but non-heated NHS did not trigger the neutrophil respiratory burst.

\section{Neutrophil adhesion}

The NHS-mediated amplification of the IgG-induced CL response originated mainly from adheredneutrophils, since the major part of the response remained when the non-adherent cells were rinsed off the surfaces. In order to elucidate if the oxygen radical production was correlated to the number of adherent neutrophils, an adhesion study was performed. Noncoated hydrophobic and hydrophilic surfaces attracted similar numbers of cells. IgG-coated hydrophobic surfaces attracted approximately 30\% more neutrophils with NHS present than the NHS-free surfaces (Figure 5). Heat-inactivation of NHS did not affect the adhesion to 
hydrophobic glass. It was found that IgG-coating on hydrophilic glass, with or without added NHS, had no effect on the number of adhered cells. Consequently, no direct correlation was found between the number of adherent neutrophils and the generation of oxygen metabolites. Morphological studies of Giemsa or bodipy-phallacidin stained neutrophils revealed similar results regarding the number of adhered cells, however showed that cells on both non- and IgG-coated (with or without NHS) surfaces displayed a more spread out morphology than cells treated with NHS only (Figure 6). Role of F-actin

The spreading and movement of neutrophils on a surface is highly dependent on a dynamic actin cytoskeleton, including reversible polymerization of actin filaments. We found that neutrophils spread out through an extensive actin polymerization in protruding lamellipodia and pseudopodia on IgGcoated hydrophobic surfaces, especially when NHS was present. Although a hydrophobic surface exposed to NHS supported the adhesion of neutrophils, the extent of spreading and actin reorganization was much more pronounced on the clean or IgG-coated surfaces (with or without NHS). Together with the CL data, these observations suggest a correlation between the cell spreading and oxidase activation. In order to explore this further, neutrophils were treated with cytochalasin B or jasplakinolide, an inhibitor and a stimulator, respectively, of actin polymerization. Although neutrophil adhesion was not affected by cytochalasin B and jasplakinolide, these drugs markedly inhibited theoxidase activation, actin polymerization and cell spreading, . The oxygen radical production on the different surfaces was totally blocked by the treatment of the neutrophils with cytochalasin B or jasplakinolide. Exposure of adherent neutrophils to cytochalasin B or jasplakinolide instantly turned off the oxygen radical production that reached the base levels within seconds. Cytochalasin B- and jasplakinolide-treated neutrophils that encountered the hydrophobic "IgG/NHSsurface" exhibited a reduced, but still obvious cell spreading. However, the organization of the actin cytoskeleton was clearly disturbed. Instead of a peripheral localization of F-actin, concentrated in pseudopodia, actin filaments were found mainly in the central parts of cytochalasin B-treated neutrophils (Figure 6). In jasplakinolide-treated neutrophils, the F-actin was localized to surface "blebs". 


\section{DISCUSSION}

The biomaterial surface wettabilityis an important factor for the activation of adhering neutrophils, probably by eliciting variation in the adsorption of proteins from complex solutions such as blood plasma. The main finding in the present work is the complement and F-actin dependent frustrated phagocytosis accompanied by a massive radical leakage on IgG-coated hydrophobic glass upon incubationin the presence of NHS. This phenomenon did not take place on any of the hydrophilic preparations.

We found that surface-bound IgG triggered a release of oxygen metabolites from neutrophil granulocytes. This is in correlation with previous in vivo ${ }^{6}$ and in vitro ${ }^{4,5}$ studies. Ellipsometric data from hydrophobic silicon surfaces showed that the IgG surface concentration was retained at a consistent level for days , but declined within hours on hydrophilic silicon. This was also consistent with our CL-data in whole blood, where the stable results on hydrophobic glass suggested a surfacemediated activation, and the scattering data on hydrophilic glass indicated a bulk-related phenomenon. From the above results we conclude that adsorbed IgG remains native enough to opsonize the model surfaces. A large IgG-binding implant area is thus likely to enhance the neutrophil respiratory burst via a Fc-receptor-mediated pathway and possibly induce frustrated phagocytosis with the release of reactive oxygen species close to the implant and surrounding tissues. It remains obscure, however, whether this frustrated phagocytosis is a positive or negative occurence for the tissue integration of a biomaterial in vivo.

The addition of NHS markedly amplified the IgG-triggered neutrophil respiratory burst in a biphasic manner. Upon the formation of an antigen-antibody complex, the classical complement activation pathway may be triggered with the subsequent formation of several components known to enhance neutrophil adhesion, chemotaxis and respiratory burst. Recently, immobilized IgG was suggested to 
initiate classical complement activation via binding of C1q from serum on methylated silicon. ${ }^{38,39}$ Both C1q and IgG can be detected by antibodies on IgG-coated hydrophobic silicon surfaces during serum incubations for up to five minutes. C3 is detected by antibodies on these surfaces for at least an hour. It has been proposed that C1q triggers the neutrophil superoxide production by a unique CD18dependent mechanism. ${ }^{40}$ The role of $\mathrm{C} 1 \mathrm{q}$ and the classical complement activation pathway were supported by the observation that the IgG-triggered oxidative response was amplified upon reconstitution of C1q to the C1q-depleted serum. However, the activation of the classical complement activation pathway can not be the only explanation for the observed CL response, since the addition of $\mathrm{C} 1 \mathrm{q}$ to the depleted serum was not a prerequisite for the amplification to occur. Also, regardless of IgG coating, a substantial radical production was observed in hydrophobic tubes when heatinactivated NHS was used, despite the fact that $\mathrm{C} 1 \mathrm{q}$ is thermolabile. ${ }^{32}$ Consequently, the role of $\mathrm{C} 1 \mathrm{q}$ and the classical activation pathway needs further investigation. Since factor B-depleted serum evoked a response similar to that of NHS after exposure to IgG-coated hydrophobic surfaces, we suggest that the alternative complement activation pathway plays a minor role for the rapid amplification of the IgG-triggered neutrophil respiratory burst.

Results from experiments with C3-depleted serum and data from Tengvall et al. (1996 and 1997) ${ }^{38,39}$ support the hypothesis that C3 deposits on adsorbed IgG, thereby promoting further complement activation and/or amplification of the neutrophil adhesion and the respiratory burst. In another study, the presence of C3 in NHS was shown to be important for the neutrophil adhesion to polymer surfaces. ${ }^{41}$ It was also demonstrated that solid surfaces, like mercaptoglycerol-modified gold, may cause a spontaneous complement activation through the increased production of iC3b and the membrane attack complex (MAC), as well as elevated deposition of C3b during incubation in NHS. ${ }^{38 \text {, }}$ 39, 42 Fragments of C3b and iC3b that were bound to materials, e.g. latex, have in other studies been suggested to increase neutrophil oxygen consumption. ${ }^{43}$ Karlsson et al. (1996) found that C3 adhered to hydrophobic surfaces, and this was accompanied by an increased respiratory burst and elevated expression of the surface integrin CD11b/CD18 (CR3) in neutrophils. ${ }^{30}$ Also, a priming phenomenon 
due to immobilization of C3 was observed, possibly due to the release of the chemotactic complement factor C5a from the hydrophobic surface. Yet another team, that worked with polystyrene-associated monomeric IgG, found attenuation of the neutrophil HOCl-generation and collagenase activation upon exposure to NHS. This was accompanied by an extracellular release of azurophilic MPO and cathepsin G. ${ }^{44}$ These effects were not seen when complement-inactivated sera were used instead of NHS and the authors suggested that C3-fixation by IgG was the primary cause for these events.

CR3 has been suggested to enhance Fc $\gamma$ R-mediated signal transduction and adhesion of neutrophils. ${ }^{45}$ The blocking of CR3 in this study with the P1-peptide reduced the CL response, suggesting that iC3b was directly involved in the neutrophil radical generation. We know from the literature that iC3b is incapable of creating a respiratory burst by itself, but in combination with stimulation of neutrophil Fc $\gamma$ RIII, a synergistic enhancement of the neutrophil oxygen radical production occurs. ${ }^{46}$ In order to show such a synergistic receptor effect in our system it is necessary to block both types of neutrophil receptors. Further testing is also required to elucidate the role of CR1 stimulation through surfacebound C3b since C3b is a bivalent molecule ${ }^{47}$ and IgG has specific attachment sites for C3b. ${ }^{48}$ The comparably low responses on the hydrophilic surfaces probably was due to the low protein retaining capacity of the hydrophilic surfaces; i.e. IgG was probably removed by the serum from the surface out to the liquid phase.

The presence of NHS alone, without surface-bound IgG, down-regulated or had no effect on the neutrophil respiratory burst. In contrast, upon exposure of clean hydrophobic surfaces to heatinactivated serum the radical generation was more than doubled, but almost totally quenched in the hydrophilic tubes (Figure 1). Therefore, it should be stressed that even though heat-inactivation of NHS inhibits the complement cascade, several soluble complement components are not thermolabile. For instance, components C3a, C4a and C5a have high degree of $\alpha$-helical conformation and each contains three disulfide bridges. Consequently, they resists the heat treatment ( $56^{\circ} \mathrm{C}$ for 30 minutes) used in this study. ${ }^{49}$ However, in this study we found that liberation of C5a from the IgG-coated 
surfaces, subsequently followed by neutrophil priming, could not be the only explanation for the very potent radical responses observed in the presence of either normal or heat-inactivated serum, since the effect of CD88-blocking was moderate. Yet another explanation is the heating process of the NHS that, theoretically, could result in the formation of various activating protein residues and aggregates, as well as providing the physiological temperature range for a limited time, where the opsonising component iC3b is known to spontaneously form in isolated serum due to the autodegradation of C3. Furthermore, a complex medium such as NHS could of course activate the neutrophils through surface activation of other plasma protein systems than complement.

The neutrophil spreading and migration over a surface is accompanied by a cyclic regulation of integrin adhesivity and actin polymerization. In this study, we also demonstrated a correlation between the extent of cell spreading and the amount of oxygen radicals that was produced. The disturbance of actin dynamics by pre-treatment of neutrophils with cytochalasin B or jasplakinolide showed no influence on the number of adherent cells on IgG/NHS-surfaces, but dramatically inhibited cell spreading, pseudopodia formation and the production of oxygen radicals. This probably reflects inhibition of the actin-dependent clustering of integrins, formation of adhesion sites and associated assembly of oxidase components. The introduction of cytochalasin B or jasplakinolide to adhered and oxygen radical producing neutrophils induced instantaneous reorganization of the actin cytoskeleton and turn-off of the respiratory burst. However, the adhesion and spreading on IgG/NHS-surfaces were more or less unaffected. Liu et al. (1997) reported a similar effect on the respiratory burst when cytochalasin B was added to adhered neutrophils, although they interpreted the oxygen radical turnoff as an indication of dependency of cell adhesion for radical production. ${ }^{5}$ We suggest that a continuos interaction between integrins and the actin cytoskeleton plays a central role for the generation of intracellular signals in adhering cells and is a prerequisite for a maintained respiratory burst. In correlation, Zhou and Brown (1994) have shown that cytochalasin B counteracts the synergism between Fc-receptors and complement receptors for the activation of the NADPH- 
oxidase. $^{46}$ They suggest that ligation of CR3 stimulates the association of Fc $\gamma$ RII with the actin cytoskeleton in the adherent neutrophils, leading to an enhanced respiratory burst.

We conclude that adsorbed IgG and the associated complement activation involving C1q, C3 and C5a in NHS, amplifies the neutrophil inflammatory responses, particularly on hydrophobic surfaces. The increased respiratory burst is accompanied with neutrophil spreading and extracellular release of oxygen radicals and is regulated by a dynamic actin cytoskeleton.

\section{ACKNOWLEDGEMENTS}

Ms Agneta Askendal, Dr. Stefan Zalavary, Mrs Kristina Orselius and Mr Mats Wolving are acknowledged for support. This study was financed by the Swedish Biomaterials Consortium funded by the Swedish National Foundation for Strategic Research, SSF, and by grant number 19X-12668 from the Swedish Medical Research Council.

\section{REFERENCES}

1. J. D. Andrade and V.L. Hlady, "Plasma protein adsorption: The big twelve,” Ann. NY Acad. Sci., 516, 158172 (1987).

2. H. Elwing, P. Tengvall, A. Askendal and I. Lundström, "'Lens-on-surface`: a versatile method for investigation of plasma protein exchange reactions on solid surfaces," in The Vroman Effect, C. H. Bamford, S. L. Cooper and T. Tsuruta (eds.), VSP, Utrecht, The Netherlands, 1992, pp. 85-93.

3. L. Tang and J. W. Eaton, "Fibrin(ogen) mediates acute inflammatory responses to biomaterials," J. Exp. Med. 178, 2147-2156 (1993).

4. L. Tang, A. H. Lucas and J. W. Eaton, "Inflammatory responses to implanted polymeric biomaterials: Role of surface-adsorbed immunoglobulin G,” J. Lab. Clin. Med. 122, 292-300 (1993).

5. L. Liu, H. Elwing, A. Karlsson, G. Nimeri and C. Dahlgren, "Surface-related triggering of the neutrophil respiratory burst; characterization of the response induced by IgG adsorbed to hydrophilic and hydrophobic glass surfaces,” Clin. Exp. Immunol. 109, 204-10 (1997). 
6. M. Källtorp, A. Askendal, P. Thomsen, and P. Tengvall, "Inflammatory cell recruitment, distribution and chemiluminescence response at IgG precoated- and thiolfunctionalized gold surfaces,” J. Biomed. Mater. Res. 47, 251-259 (1999).

7. D. E. Chenoweth, "Complement activation during hemodialysis: Clinical observations, proposed mechanisms, and theoretical implications,” Artif. Organs 8, 281-287 (1984).

8. A. K. Cheung, C. J. Parker, L. Wilcox and J. Janatova, "Activation of the alternative pathway of complement by cellulosic hemodialysis membranes,” Kidney Int. 36, 257-265 (1989).

9. A. D. Shepard, J. A. Gelfand, A. D. Callow and T. F. O`Donnel, “Complement activation by synthetic vascular prostheses,” J. Vasc. Surg. 1, 829-838 (1984).

10. J. C. A. De Mol Van Otterlo, J. H. Van Bockel, E. D. Ponfort, E. J. P. Brommer, J. Hermans and M. R. Daha, "The effects of aortic reconstruction and collagen impregnation of Dacron prostheses on the complement system,” J. Vasc. Surg. 16, 774-783 (1992).

11. R. Depisch, H. Göhl, E. Ritz and G. M. Hänsch, “Complement Activation on Artificial Surfaces in Biomedical Therapies,” in The Complement system, K. Rother, G. O. Till and G. M. Hänsch, Springer Verlag, Berlin Heidelberg, 1998, pp. 487-504.

12. T. Kinoshita, “Biology of complement: the overture,” Immunol. Today 12, 291-295 (1991).

13. J. Janatova, A. K. Cheung and C. J. Parker, "Biomedical polymers differ in their capacity to activate complement,” Complement Inflammation 8, 61-69 (1991).

14. C. Eriksson and H. Nygren, “The initial reactions of graphite and gold with blood,” J. Biomed. Mater. Res. 37, 130-136 (1997).

15. C. Eriksson, E. Blomberg, P. Claesson and H. Nygren, "Reactions of two hydrophilic surfaces with detergents, protein and whole human blood,” Colloids. Surf. B 9, 67-79 (1997).

16. H. Nygren, C. Eriksson and J. Lausmaa, "Adhesion of platelets and polymorphonuclear granulocyte cells at $\mathrm{TiO}_{2}$ surfaces,” J. Lab. Clin. Med. 1, 35-46 (1997).

17. M. M. Frank and L. F. Fries, “The role of complement in inflammation and phagocytosis,” Immunol. Today 12, 322-326 (1991).

18. H.Y.K. Chuang, S.F. Mohammad and R.F. Mason, "Prostacyclin $\left(\mathrm{PGI}_{2}\right)$ inhibits the enhancement of granulocyte adhesion to cuprophane induced by Immunoglobulin G,” Thromb. Res. 19, 1-9 (1980).

19. L. Liu and H. Elwing, "Complement activation on solid surfaces as determined by C3 deposition and hemolytic consumption,” J. Biomed. Mater. Res. 28, 767-773 (1994). 
20. J. I. Gallin, “The neutrophil,” in Immunological diseases (4th ed.), M. Sander, D. W. Talmore, M. M. Frank, K. F. Austen and H. N. Claman (eds.) Little, Brown and Co., Rock, pp. 737-788, 1988.

21. D. W. Sawyer, G. R. Donowitz and G. L. Mandell, "Polymorphonuclear neutrophils: An effective antimicrobical force,” Rev. Infect. Dis. 11, 1532-1544 (1989).

22. C. Dahlgren, P. Follin, A. Johansson, R. Lock, H. Lundqvist and Å. Walan, “Chemiluminescence as a means of following the function of phagocytic cells,” Trends Photochem. Photobiol. 2, 427-443 (1991).

23. F. A. Al Mohanna and M. B. Hallet, “Actin polymerization modifies stimulus-oxidase coupling in rat neutrophils,” Biochim. Biophys. Acta 927, 366-371 (1987).

24. J. Norgauer, E. Kownatzki, R. Seifert and K. Aktories, "Botulinum C2 toxin ADP-ribosylates actin and enhances $\mathrm{O}_{2}^{-}$production and secretion but inhibits migration of activated human neutrophils,” J. Clin. Invest. 82, 1376-1382 (1988).

25. T. Bengtsson, C. Dahlgren, O. Stendahl, T. Andersson, “Actin assembly and regulation of neutrophil function: Effects of cytochalasin $\mathrm{B}$ and tetracaine on chemotactic peptide-induced $\mathrm{O}_{2}^{-}$production and degranulation,” J. Leukoc. Biol. 49, 236-44 (1991).

26. T. Morimatsu, A. Kawagoshi, K. Yoshida and M. Tamura, "Actin enhances the activation of neutrophil NADPH oxidase in a cell-free system," Biochem. Biophys.Res. Commun. 230, 206-210 (1997).

27. S. R. Yan, L. Fumagalli, S. Dusi, G. Berton, "X-100-insoluble, cytoskeletal fraction of beta 2 integrins, NADPH oxidase components, tyrosine phosphorylated proteins, and the protein tyrosine kinase p58fgr in human neutrophils adherent to fibrinogen, " J. Leukoc. Biol. 58, 595-606 (1995).

28. W. Kern and D. A. Puotinen, "Cleaning solutions based on hydrogen peroxide for use in silicon semiconductor technology,” RCA Rev. 29, 187-206 (1970).

29. D. A. Peters and C. A. Deckert, "Removal of Photoresist Film Residues from Wafer Surfaces,” J. Elechtrochem. Soc. 126, 883-886 (1979).

30. C. Karlsson, H. Nygren and M. Braide, "Exposure of blood to biomaterial surfaces liberates substances that activate polymorphonuclear granulocytes,” J. Lab. Clin. Med. 128, 496-505 (1996).

31. A. Böyum, "Isolation of mononuclear cells and granulocytes from human blood. Isolation of mononuclear cells by one centrifugation and of granulocytes by combining centrifugation and sedimentation at 1 x $g$,” Scand. J. Clin. Lab. Invest. 21, 77-89 (1968). 
32. W. P. Kolb, L. M. Kolb and R. P. Eckhard, “C1q: Isolation from human serum in high yield by affinity chromatography and development of a highly sensitive hemolytic assay,” J. Immunol. 122, 2103-2111 (1979).

33. S. Diamond, C. S. Johnston, M. L. Dustin, P. McCaffery and T. A. Springer, "Differential effects on leucocyte functions of CD11a, CD11b, and CD18 mAb,” in White cell differentiation antigens; Leukocyte typing IV W. Knapp (ed.), Oxford University Press, Oxford, New York, 1989, pp. 570-574.

34. C. Weber and T. A. Springer, "Neutrophil accumulation on activated, surface-adherent platelets in flow is mediated by interaction of Mac-1 with fibrinogen bound to alphaIIbbeta3 and stimulated by plateletactivating factor,” J. Clin. Invest. 100, 2085-93 (1997).

35. T. Bengtsson and M. Grenegård, "Platelets amplify chemotactic peptide-induced changes in F-actin and calcium in human neutrophils,” Eur. J. Cell. Biol. 63, 345-349 (1994).

36. A. A. Vaporciyan, M. L. Jones and P.A. Ward, "Rapid analysis of leukocyte-endothelial adhesion," J. Immunol. Methods 159, 93-100 (1993).

37. A. Papayianni, C. N. Serhan and H. R. Brady, "Lipoxin $A_{4}$ and $B_{4}$ inhibit leukotriene-stimulated interactions of human neutrophils and endothelial cells,” J. Immunol. 156, 2264-2272 (1996).

38. P. Tengvall, A. Askendahl and I. Lundström, “Complement activation by IgG immobilized on methylated silicon,” J. Biomed. Mater. Res. 31, 305-312 (1996).

39. P. Tengvall, A. Askendal and I. Lundström, “Temporal studies on the deposition of complement on human colostrum IgA and serum IgG immobilized on methylated silicon,” J. Biomed. Mater. Res. 35, 81-92 (1997).

40. E. B. Goodman, D. C. Anderson and A. J. Tenner, "C1q triggers neutrophil superoxide production by a unique CD18-dependent mechanism,” J. Leukoc. Biol. 58, 168-176 (1995).

41. G. A. Herzlinger and R.D. Cumming, "Role of complement in cell adhesion to polymer blood contact surfaces,” Trans. Am. Soc. Artif. Organs 26, 165-171 (1980).

42. L. Liu and H. Elwing, “Complement activation on thiol-modified gold surfaces,” J. Biomed. Mater. Res. 30, 535-541 (1996).

43. M. Hoogerwerf, R. S. Weening, C. E. Hack and D. Roos, “Complement fragments C3b and iC3b coupled to latex induce a respiratory burst in human neutrophils,” Mol. Immunol. 27, 159-167 (1990).

44. W. W. Chatham and W. D. (Jr.) Blackburn, "Fixation of C3 to IgG attenuates neutrophil HOCl generation and collagenase activation,” J. Immunol. 151, 949-958 (1993). 
45. T. Kusunoki, S. Tsuruta, H. Higashi, S. Hosoi, D. Hata, K. Sugie, M. Mayumi and H. Mikawa, "Involvement of CD11b/CD18 in enhanced neutrophil adhesion by Fcy receptor stimulation,” J. Leukoc. Biol. 55, 735-742 (1994).

46. M. Zhou and E. J. Brown, "CR3 (Mac-1, $\alpha_{\mathrm{M}} \beta_{2}$, CD11b/CD18) and Fc $\gamma$ RIII cooperate in generation of a neutrophil respiratory burst: Requirement for FcyRIII and tyrosine phosphorylation,” J. Cell. Biol. 125, 1407-1416 (1994).

47. A. Erdei, G. Füst and J. Gergely, "The role of C3 in the immune response," Immunol. Today 12, 332-337 (1991).

48. M. K. Pangburn, "Alternative pathway: Activation and regulation," in The Complement System (2nd ed.) K. Rother, G. O. Till and G. M. Hänsch (eds.), Springer-Verlag, Berlin Heidelberg, pp. 93-115, 1998.

49. I. M. Goldstein, "Complement: Biologically active products," in Inflammation: Basic principles and clinical correlates (2nd ed.) J. I. Gallin, I. M. Goldstein and R. Snyderman (eds.), Raven Press, Ltd., New York, pp. 63-80, (1992). 


\section{Table 1: Effects of immobilized IgG on the radical production in diluted whole blood}

Luminol-amplified CL was measured in hydrophobic and hydrophilic glass tubes, with or without IgG-precoating, at $37{ }^{\circ} \mathrm{C}$ for 30 minutes in a Multi-Biolumat. $4 \mathrm{U} / \mathrm{ml}$ horseradish peroxidase was added. The total amount of counts per minute was observed and expressed as per cent of the response at non-coated surfaces \pm standard error of the mean (SEM). The experiments were performed in duplicate with three or four donors.

Figure 1. Effect of surface-bound immunoglobulin G (IgG) on the neutrophil respiratory burst in the absence or presence of normal human serum (NHS). Luminolamplified chemiluminescence (CL) was measured in (A) hydrophobic or (B) hydrophilic glass test tubes with or without $5 \%(\mathrm{v} / \mathrm{v})$ normal or heat-inactivated serum and $1 \times 10^{6}$ neutrophils per ml KRG at $37{ }^{\circ} \mathrm{C}$ for 30 minutes. Extra peroxidase was added (4U horseradish peroxidase per $\mathrm{ml}$ ). The results, based on the total amount of counts per minute, are expressed as percent of control (non-coated hydrophobic or hydrophilic glass) and represents the mean values from separate experiments with 12 (A) or 9 (B) donors run at least in duplicate. Bars indicates the standard errors of the means (SEM). For absolute values of the controls, see text. On hydrophobic glass, "IgG and NHS" $(\mathrm{p}<0.001)$, "IgG and Heatinactivated serum" $(\mathrm{p}<0.001)$ and "Heat-inactivated NHS" $(\mathrm{p}<0.01)$, and on hydrophilic surfaces “NHS” ( $\mathrm{p}<0.01)$, “Heat-inactivated NHS” ( $\mathrm{p}<0.001)$ and "IgG and Heat-inactivated serum” ( $<<0.001)$, were found to differ significantly from control cells.

Figure 2. Chemiluminescence (CL) profiles that show representative kinetics for the actual time traces of CL from $1 \times 10^{6}$ neutrophils/ml in KRG during 30 minutes of incubation in $37^{\circ} \mathrm{C}$ with HRP $(4 \mathrm{U} / \mathrm{ml})$, luminol $\left(10^{-5} \mathrm{M}\right)$ and NHS (5\%) or heat-inactivated NHS (5\%) added to IgG-coated hydrophobic or hydrophilic glass tubes. An instantaneous radical turnoff occurs when jasplakinolide $(5 \mu \mathrm{M})$ or cytochalasin B $(12.5 \mu \mathrm{M})$ are added (illustrated 
with the CL-profile after addition of jasplakinolide at time $=15$ minutes). The CL was registered as counts per minute (CPM) and the neutrophils were isolated from the same donor (exept for the cells treated with jasplakinolide).

\section{Figure 3. Effects of surface-bound Immunoglobulin G (IgG) on the neutrophil} respiratory burst in the absence or presence of C3-depleted serum. Luminol-amplified chemiluminescence (CL) was measured in hydrophobic glass test tubes with 5\% (v/v) C3depleted serum and $1 \times 10^{6}$ neutrophils per $\mathrm{ml}$ at $37^{\circ} \mathrm{C}$ for 30 minutes. Extra peroxidase was added ( $4 \mathrm{U}$ horseradish peroxidase per ml). The results, based on the total amount of counts per minute, are expressed as percent of the response at non-coated hydrophobic surfaces and represents the mean value of separate experiments with 4 donors run in duplicate. Bars indicate the standard errors of the means (SEM). "IgG/C3-depleted serum" differs significantly from “IgG/C3-depleted serum + C3” ( $<<0.05)$.

Figure 4. (A) Reconstitution with C1q $(180 \mu \mathrm{g} / \mathrm{l})$ in the C1q-depleted serum further potentiates the total IgG-triggered neutrophil respiratory burst on hydrophobic glass $(\mathrm{p}<0.05)$. (B) Blocking of the CD88 through 30 minutes of pre-incubation at $4^{\circ} \mathrm{C}$ with $10 \mu \mathrm{g} / \mathrm{ml}$ of the azide free MCA $1283 \mathrm{XZ}$ antibody significantly $(\mathrm{p}<0.05)$ lowers the hydrophobic "IgG/NHS-effect”. Similar incubation with the control antibody RDI-MSIGG2A-X showed no effect on the luminol-amplified chemiluminescence $(C L)$ response. Counts per minute (CPM) data represents the total CL from $1 \mathrm{x}$ $10^{6}$ neutrophils per $\mathrm{ml}$, measured for 30 minutes at $37{ }^{\circ} \mathrm{C}$ in hydrophobic tubes with $4 \mathrm{U} / \mathrm{ml}$ horseradish peroxidase and 5\% (v/v) serum added. Experiments were performed in duplicate with cells from three different donors. Bars indicates the standard errors of the means (SEM).

Figure 5. Neutrophil adhesion. Fluorescence from BCECF-labelled, adhered neutrophils (rinsed samples) expressed as percent of the response on non-coated hydrophobic surfaces (control). The fluorescence from non-rinsed (free and adherent cells) samples was 336\% ( \pm 
23\%). The autofluorescence from non-labelled cells was routinely subtracted. The experiments were performed in triplicate with neutrophils from four donors. Bars indicate the SEM. "IgG and NHS" was found to adhere significantly $(\mathrm{p}<0.05)$ more cells than all other coatings. No other significant differences were found. NHS denotes the presence of $5 \%$ normal human serum, and IgG indicates IgG-coated hydrophobic glass. Heat-inactivated serum displayed similar results as NHS.

Figure 6. Neutrophils on hydrophobic glass with various protein coatings after 30 minutes of incubation in $37^{\circ} \mathrm{C}$ KRG. The neutrophils were fixed (4\% paraformaldehyde) and F-actin was stained ( $0.6 \mu \mathrm{g} / \mathrm{ml}$ bodipy-phallacidin, $100 \mu \mathrm{g} / \mathrm{ml}$ lysophosphatidylcholine), studied and documented with a Sarastro 1000 confocal laser scanning microscope with a Nikon x 100 (1.4 N.A.) objective. Control indicates clean hydrophobic glass, NHS denotes presence of 5\% normal human serum, and IgG indicates IgG-coated hydrophobic glass. Heat-inactivated serum gave rise to morphology similar to that with NHS. 
“Complement activation on immunoglobulin G-coated hydrophobic surfaces...”

Table 1: Effects of immobilized IgG on the radical production in diluted whole blood

\begin{tabular}{|c|c|c|c|}
\hline & No dilution & $50 \%$ blood & $25 \%$ blood \\
\hline Hydrophobic glass $(n=4)$ & $995 \%( \pm 302 \%)$ & $1220 \%( \pm 330 \%)$ & $856 \%( \pm 389 \%)$ \\
\hline Hydrophilic glass $(n=3)$ & $669 \%( \pm 148 \%)$ & $352 \%( \pm 44,1 \%)$ & $168 \%( \pm 45,4 \%)$ \\
\hline
\end{tabular}


Figure 1

\section{A: Hydrophobic glass}

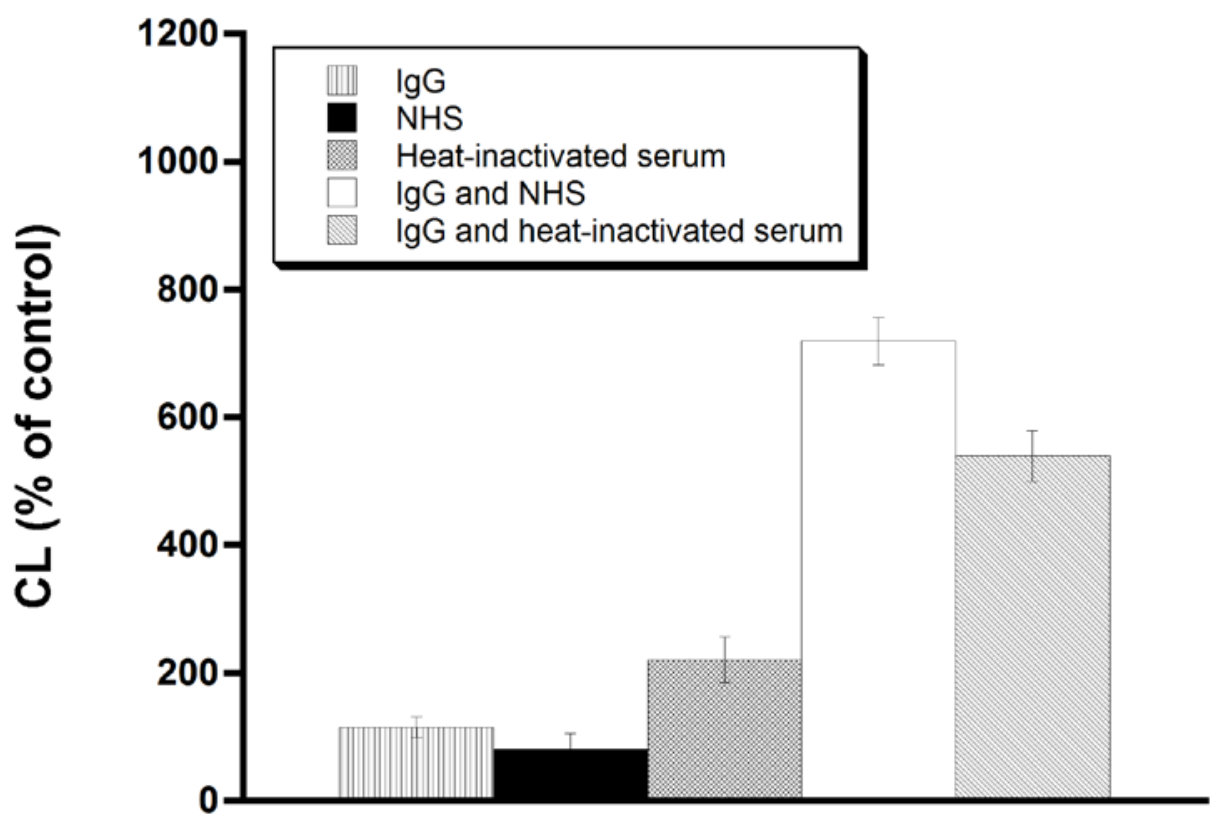




\section{B: Hydrophilic glass}

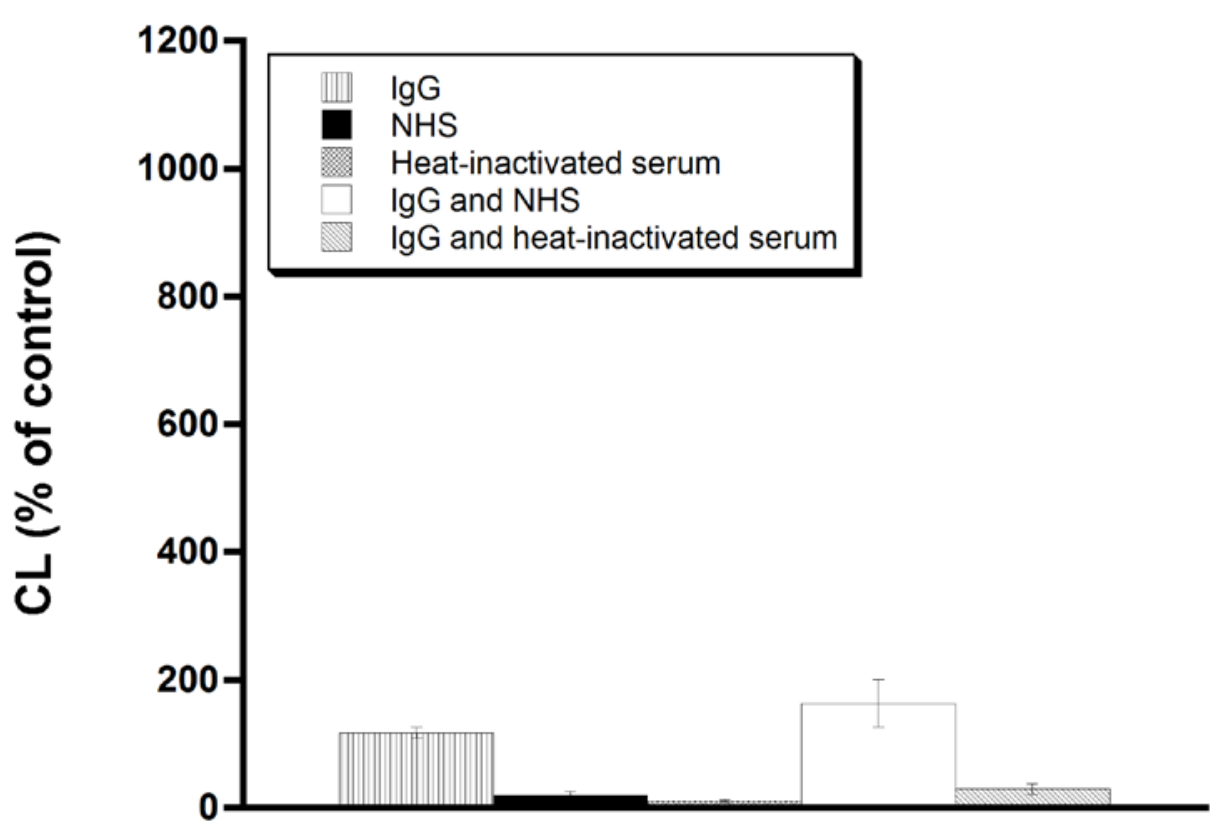


Figure 2

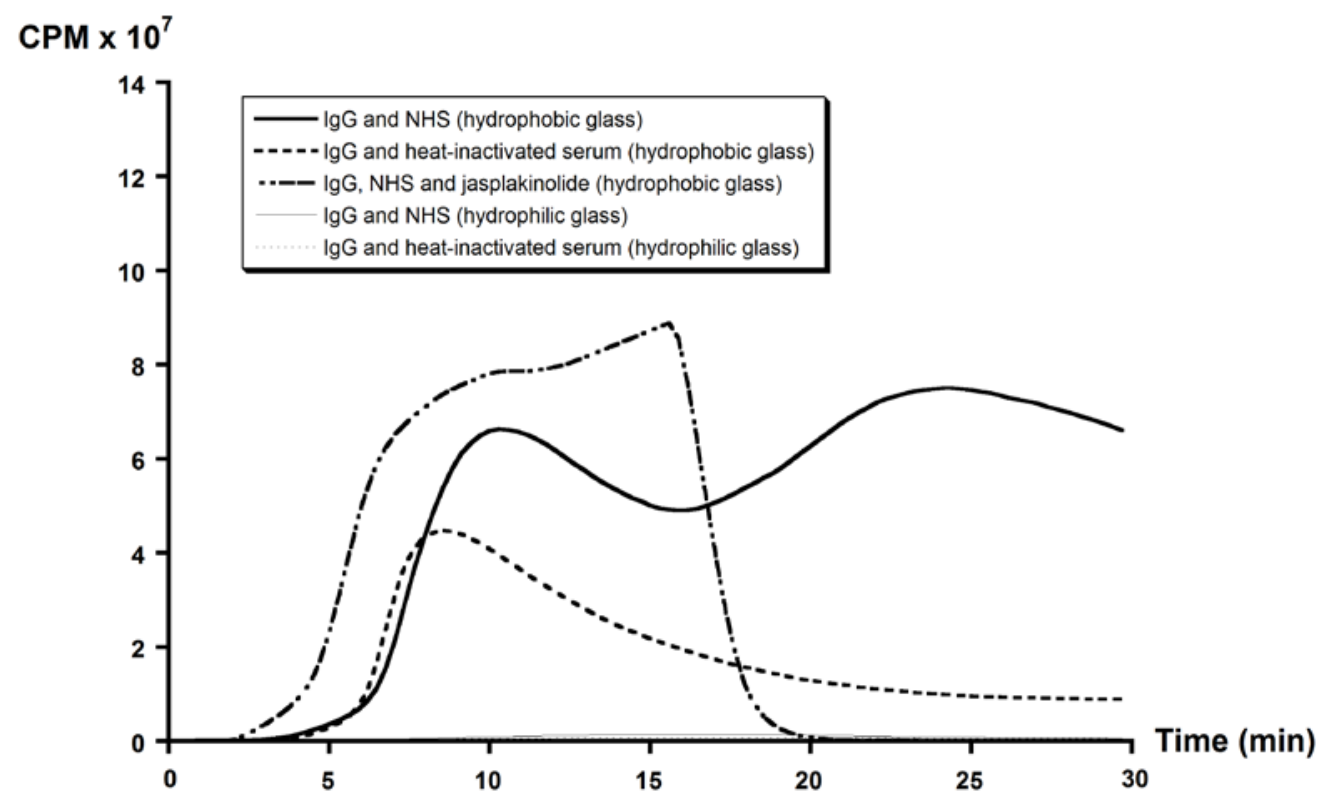


Figure 3

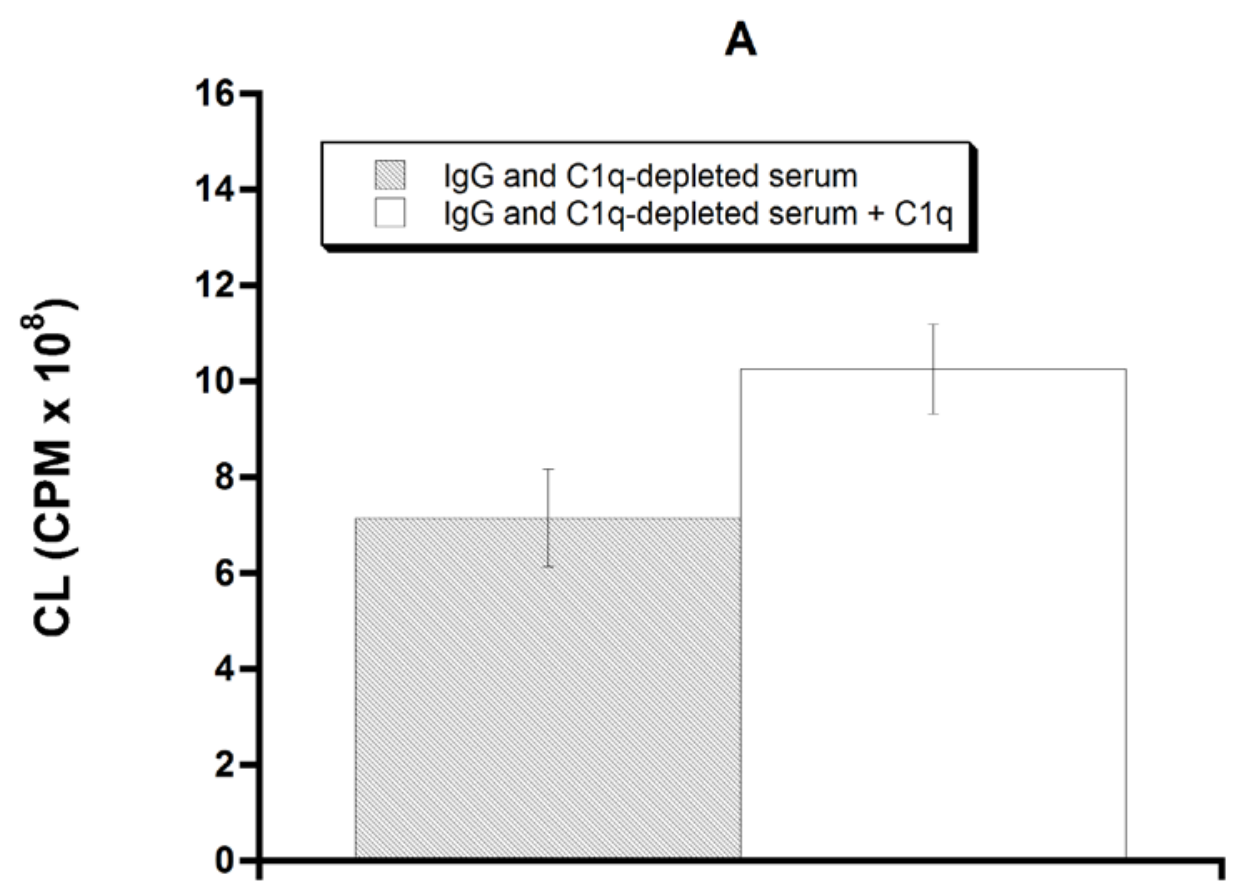

B

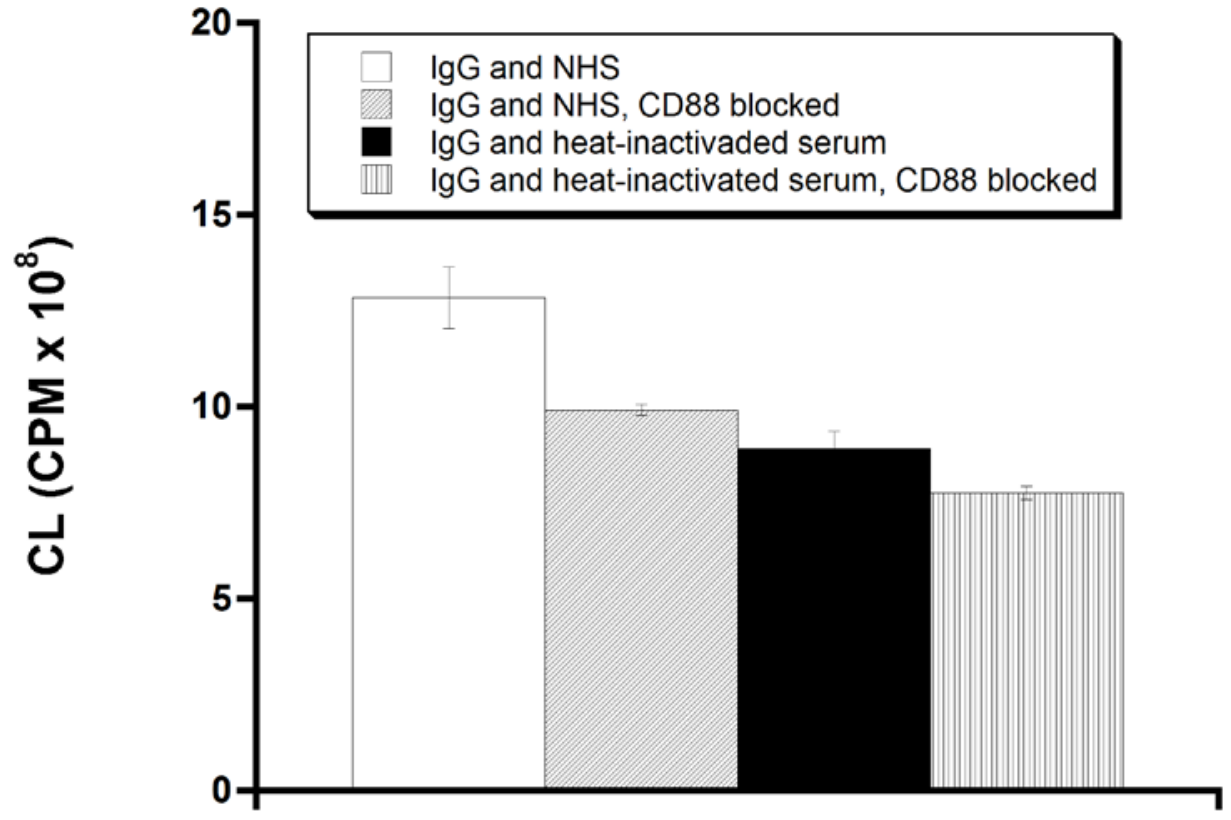


Figure 4

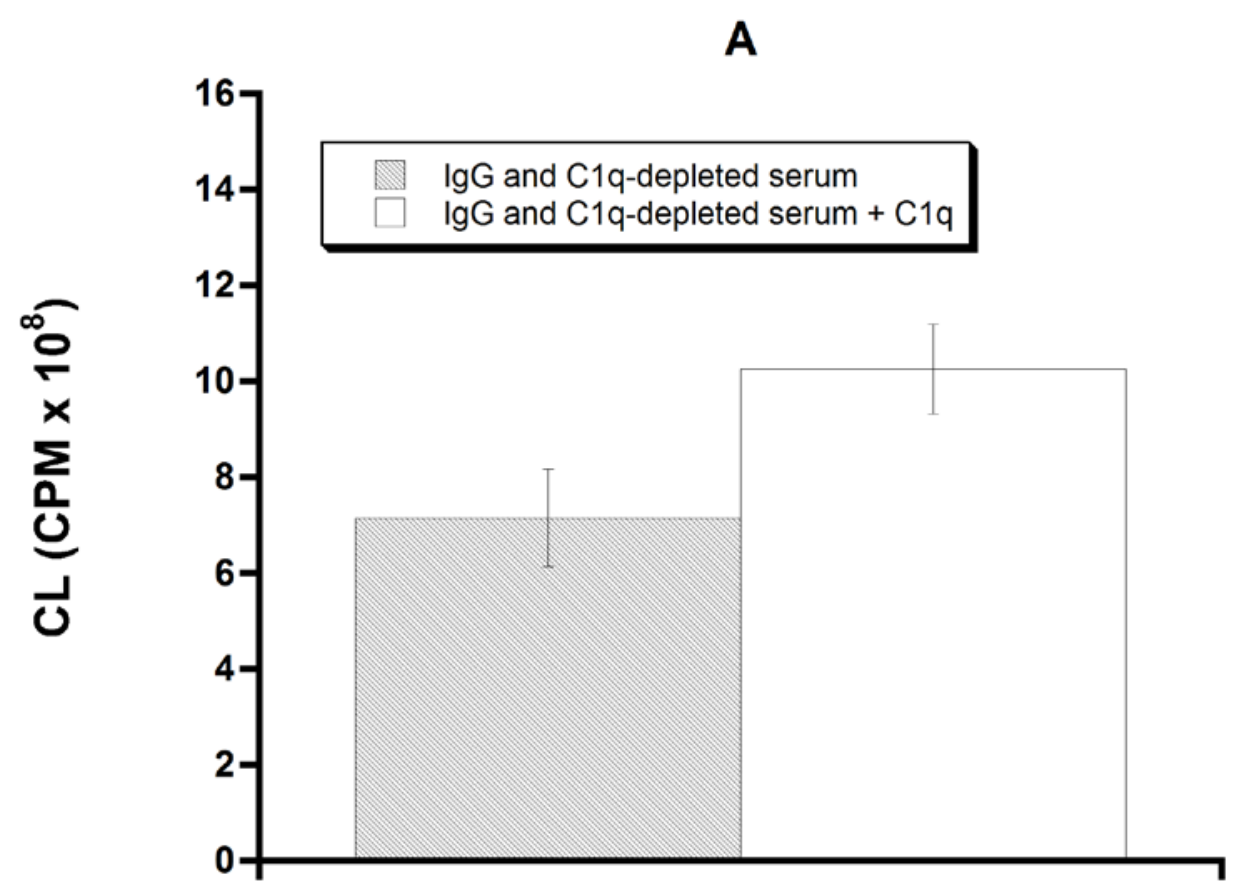

B

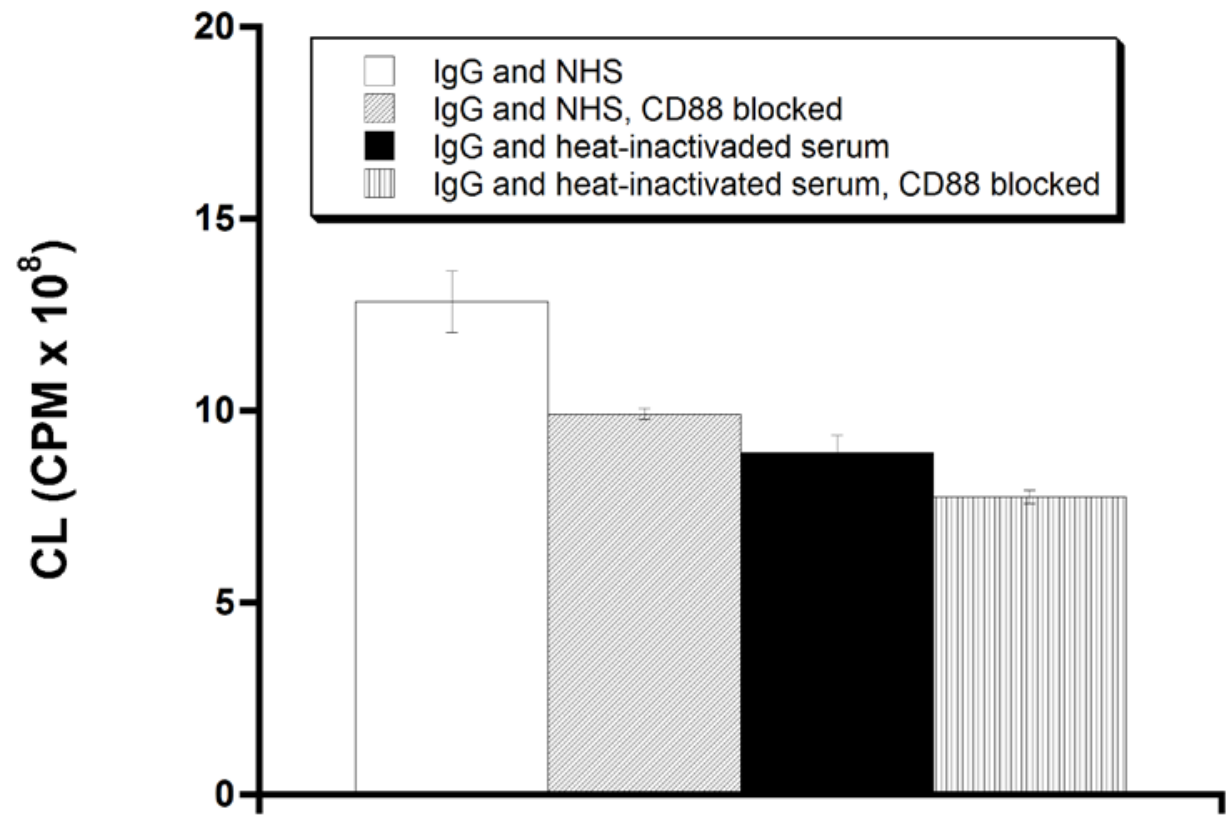


Figure 5

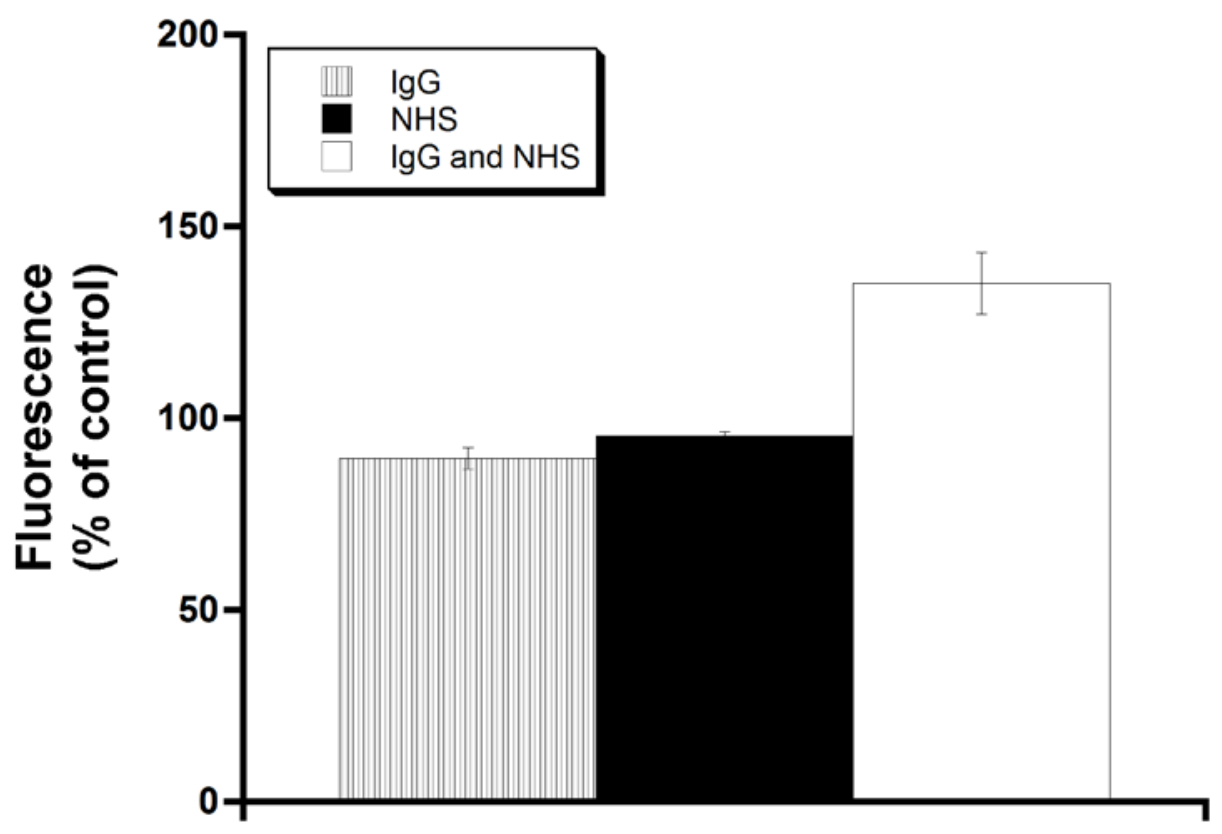


Figure 6

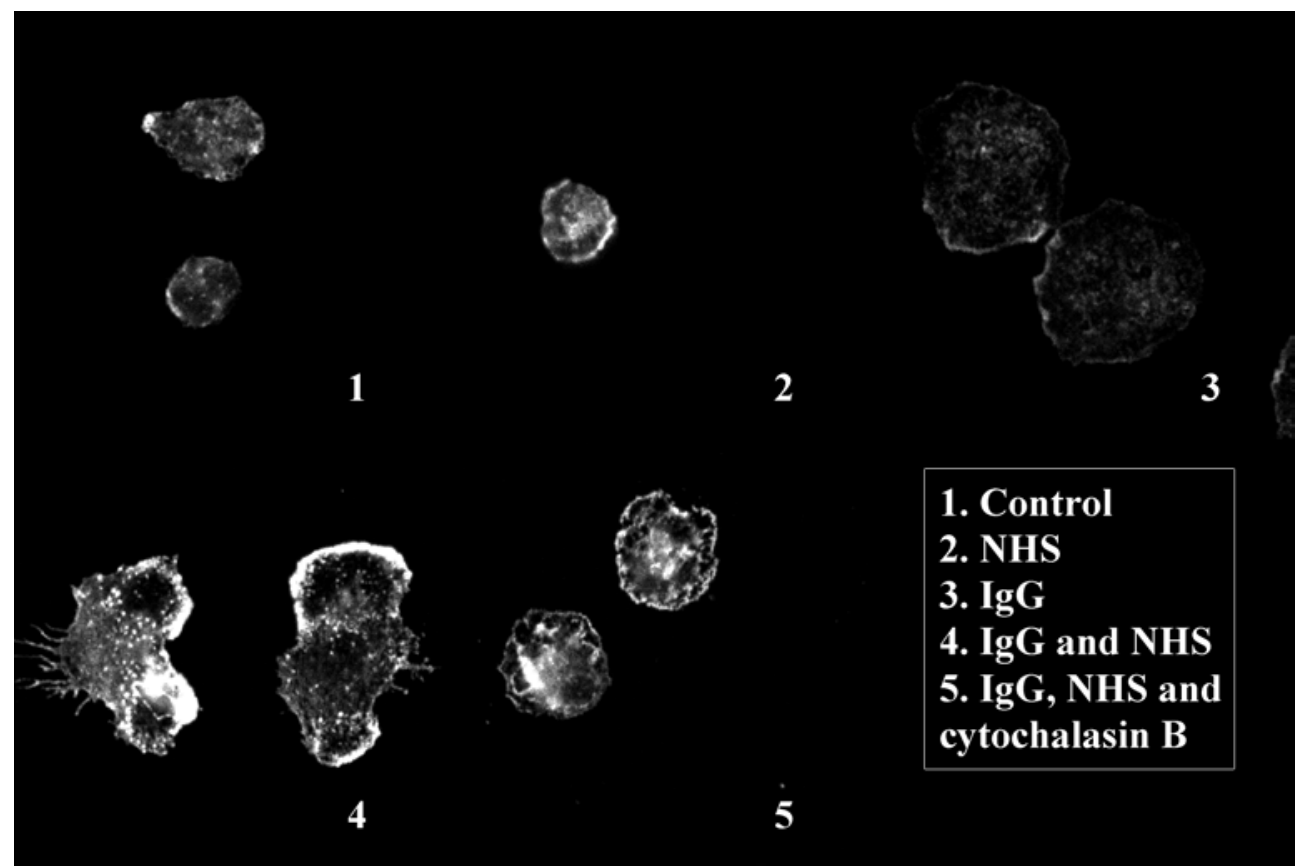

